PROCEEDINGS OF THE

AMERICAN MATHEMATICAL SOCIETY

Volume 135, Number 12, December 2007, Pages 4031-4037

S 0002-9939(07)08924-1

Article electronically published on August 29, 2007

\title{
A CHARACTERIZATION OF FIBRANT SEGAL CATEGORIES
}

\author{
JULIA E. BERGNER
}

(Communicated by Paul Goerss)

\begin{abstract}
In this note we prove that Reedy fibrant Segal categories are fibrant objects in the model category structure $\mathcal{S C}_{c}$. Combining this result with a previous one, we thus have that the fibrant objects are precisely the Reedy fibrant Segal categories. We also show that the analogous result holds for Segal categories that are fibrant in the projective model structure on simplicial spaces, considered as objects in the model structure $\mathcal{S C}_{f}$.
\end{abstract}

\section{INTRODUCTION}

Segal categories are simplicial spaces which are meant to look like simplicial categories, but their morphisms are only associative up to (higher) homotopy. They were first described by Dwyer, Kan and Smith, who called them special $\boldsymbol{\Delta}^{o p}$-spaces 3]. They have since been generalized to the notion of Segal $n$-categories, variants of which have been studied as models for weak $n$-categories by Hirschowitz and Simpson [7, 13] and Tamsamani [14. Like simplicial categories, they can be considered as models for homotopy theories [2, 8.6].

In any model category structure, it is useful to have a precise description of the fibrant and cofibrant objects, since these are used to define the homotopy category. Furthermore, various constructions, such as that of mapping spaces, are only homotopy invariant when the objects involved are both fibrant and cofibrant. In the first of the model structures which we will consider, $\mathcal{S} e \mathrm{C} a t_{c}$, all objects are cofibrant, but a description of the fibrant objects has not been so clear. In this note, we give a complete characterization of them.

We begin by giving some precise definitions. Let $\boldsymbol{\Delta}$ denote the cosimplicial category, or category whose objects are finite ordered sets $[n]=(0 \rightarrow 1 \rightarrow \ldots \rightarrow n)$ for $n \geq 0$ and whose morphisms are order-preserving maps between them. Then $\boldsymbol{\Delta}^{o p}$ is the opposite of this category and is called the simplicial category. Recall that a simplicial set $X$ is a functor $\boldsymbol{\Delta}^{o p} \rightarrow$ Sets. We will denote the category of simplicial sets by SSets. (In the course of this paper we will sometimes refer to simplicial sets as spaces, due to their homotopy-theoretic similarity with topological spaces [8, 3.6.7].) We denote by $\Delta[n]$ the $n$-simplex for each $n \geq 0$, by $\dot{\Delta}[n]$ its boundary, and by $V[n, k]$ the boundary with the $k$ th face removed. More details about simplicial sets can be found in [5, I]. A simplicial set $X$ is discrete if all elements of $X_{n}$ are degenerate for $n>0$. We denote by $|X|$ the topological space given by geometric realization of the simplicial set $X$ [5, I.2].

Received by the editors May 2, 2006 and, in revised form, August 30, 2006.

2000 Mathematics Subject Classification. Primary 55U35; Secondary 18 G30.

(c) 2007 American Mathematical Society Reverts to public domain 28 years from publication 
More generally, recall that a simplicial object in a category $\mathcal{C}$ is a functor $\boldsymbol{\Delta}^{o p} \rightarrow$ C. In particular, a functor $\boldsymbol{\Delta}^{o p} \rightarrow$ SSets is a simplicial space or bisimplicial set $[5$, IV]. Given a simplicial set $X$, we denote by $X^{t}$ the simplicial space such that $\left(X^{t}\right)_{0}$ is the discrete simplicial set $X_{0}$. Also, given a simplicial space $W$, we denote by $\operatorname{sk}_{n} W$ the $n$-skeleton of $W$, or the simplicial space generated by the simplices of $W$ of degree less than or equal to $n$ [11, $\S 1]$. In this paper, our primary concern is the case where the simplicial set in degree zero is discrete (or constant).

Definition 1.1. A Segal precategory is a simplicial space $X$ such that $X_{0}$ is a discrete simplicial set.

Now note that for any simplicial space $X$ there is a Segal map

$$
\varphi_{k}: X_{k} \rightarrow \underbrace{X_{1} \times_{X_{0}} \ldots \times_{X_{0}} X_{1}}_{k}
$$

for each $k \geq 2$, which we define as follows. Let $\alpha^{i}:[1] \rightarrow[n]$ be the map in $\boldsymbol{\Delta}$ such that $\alpha^{i}(0)=i$ and $\alpha^{i}(1)=i+1$, defined for each $0 \leq i \leq n-1$. We can then define the dual maps $\alpha_{i}:[n] \rightarrow[1]$ in $\boldsymbol{\Delta}^{o p}$. Given a simplicial space $X$, for each $k \geq 2$ the Segal map is defined to be the map

$$
\varphi_{k}: X_{k} \rightarrow \underbrace{X_{1} \times_{X_{0}} \cdots \times_{X_{0}} X_{1}}_{k}
$$

induced by the maps

$$
X\left(\alpha_{i}\right): X_{k} \rightarrow X_{1} .
$$

Definition 1.2. 7, §2] A Segal category $X$ is a Segal precategory such that the Segal map $\varphi_{k}$ is a weak equivalence of simplicial sets for each $k \geq 2$.

For a Segal (pre)category $X$, we will frequently refer to the discrete simplicial set $X_{0}$ as the set of "objects" of $X$.

In [2, 5.1, 7.1], we show that there exist two different model category structures on the category of all Segal precategories in which the fibrant objects are Segal categories. (We will discuss model category structures in more detail in the next section.) For the first of these structures, $\mathrm{SeCat}_{c}$, we show in [2, 5.13] that the fibrant objects are Segal categories that are fibrant in the Reedy model category structure on the category of all simplicial spaces. Here, we complete the result and show that the converse holds as well, namely, that all Reedy fibrant Segal categories are fibrant in $\mathrm{SeCat}_{c}$. Similarly, in the second model structure, $\mathcal{S} e \mathrm{C} a t_{f}$, we show that the fibrant objects are precisely the Segal categories that are fibrant in the projective model category structure on simplicial spaces.

It should be noted that these model category structures on Segal precategories fit into a chain of Quillen equivalences between various model structures. The two are Quillen equivalent to one another, as well as to a model structure on the category of simplicial categories and to Rezk's complete Segal space model structure on simplicial spaces, which Joyal and Tierney prove to be Quillen equivalent to Joyal's model structure on quasi-categories [9], 10. In doing so, they actually obtain an alternate proof of Theorem 3.2. The author's interest in comparing these model structures arose from finding models for the homotopy theory of homotopy theories, a project begun by Rezk [12]. 


\section{REVIEW OF MODEL CATEGORY STRUCTURES}

In this section, we give a brief review of model category structures. In particular, we discuss the Reedy model category structure on the category of all simplicial spaces and the model category structure $\mathcal{S} e \mathcal{C} a t_{c}$ on the category of Segal precategories.

Recall that a model category structure on a category $\mathcal{C}$ is a choice of three distinguished classes of morphisms: fibrations, cofibrations, and weak equivalences. A (co)fibration that is also a weak equivalence is an acyclic (co)fibration. With this choice of three classes of morphisms, $\mathcal{C}$ is required to satisfy five axioms MC1-MC5 which can be found in [4,3.3]. An object $X$ in a model category is fibrant if the unique map $X \rightarrow *$ to the terminal object is a fibration. Dually, $X$ is cofibrant if the unique map from the initial object $\phi \rightarrow X$ is a cofibration.

There is a model category structure on the category of simplicial sets in which the weak equivalences are the maps $f: X \rightarrow Y$ such that the induced map $|f|$ : $|X| \rightarrow|Y|$ of topological spaces is a weak homotopy equivalence [5, I.11.3]. We can then use this model category structure to define model structures on the category of simplicial spaces.

A natural choice for the weak equivalences in the category of all simplicial spaces is the class of levelwise weak equivalences of simplicial sets. Namely, given two simplicial spaces $X$ and $Y$, we define a map $f: X \rightarrow Y$ to be a weak equivalence if and only if for each $n \geq 0$, the map $f_{n}: X_{n} \rightarrow Y_{n}$ is a weak equivalence of simplicial sets.

In the Reedy model category structure on simplicial spaces [11, the weak equivalences are the levelwise weak equivalences of simplicial sets. We will denote the Reedy model structure by $\operatorname{SS}_{e t} s_{c}^{\Delta^{o p}}$. While it is defined somewhat differently, the Reedy model category structure on simplicial spaces is exactly the same as the injective model category structure on this same category, in which the cofibrations are defined to be levelwise cofibrations of simplicial sets [6, 15.8.7].

In section 4 , we will also make use of the projective model category structure $\operatorname{SSet} s_{f}{ }^{o p}$ on simplicial spaces, in which the fibrations are given by levelwise fibrations of simplicial sets [5, IX 1.4].

In [12, 7.1], Rezk defines a model category structure which is obtained by localizing $\operatorname{SSets}_{c}^{\Delta^{o p}}$ with respect to a set of maps [6, 4.1.1]. Its fibrant objects are called Segal spaces, and they satisfy two properties: they are fibrant in the Reedy model structure, and each Segal map $\varphi_{k}$ is a weak equivalence for $k \geq 2$. In particular, there is a fibrant replacement functor taking any simplicial space to a Segal space. In [2, §5], we construct a similar functor $L_{c}$ which takes a Segal precategory $X$ to a Segal space $L_{c} X$ which is also a Segal category such that $X_{0}=\left(L_{c} X\right)_{0}$.

Theorem 2.1. 2, 5.1] There is a model category structure SeCat on the category of Segal precategories with the following weak equivalences, fibrations, and cofibrations:

- Weak equivalences are the maps $f: X \rightarrow Y$ such that the induced map $L_{c} X \rightarrow L_{c} Y$ is a weak equivalence in Rezk's model structure on simplicial spaces.

- Cofibrations are the monomorphisms. (In particular, every Segal precategory is cofibrant.)

- Fibrations are the maps with the right lifting property with respect to the maps that are both cofibrations and weak equivalences. 
Corollary 2.2. [2, 5.13] Fibrant objects in SeCat are Reedy fibrant Segal categories.

We would like to prove the converse of this corollary. We begin by proving it in a more restricted setting, namely in the model category of Segal precategories which have a fixed set $\mathcal{O}$ in degree zero.

\section{Reedy fibrant Segal CAtegories}

We begin this section by characterizing the fibrant objects in the fixed object set case, and then we proceed to the more general case. We begin by briefly describing the model structure in the more restricted situation.

We show in [1, 3.9] that there is a model structure $\mathcal{S} S p_{\mathcal{O}, c}$ on the category of Segal precategories that have a fixed set $\mathcal{O}$ in degree zero and in which the weak equivalences and cofibrations are each defined levelwise. This model category can be localized with respect to a set of maps to obtain a model structure $\mathcal{L} \mathcal{S} S p_{\mathcal{O}, c}$, in which the fibrant objects are Segal categories.

Thus, we will first prove that the fibrant objects of $\mathcal{L} \mathcal{S} \mathcal{S} p_{\mathcal{O}, c}$ are precisely the Reedy fibrant Segal categories with the set $\mathcal{O}$ in degree zero. Note that any Reedy fibrant Segal precategory is a fibrant object in the appropriate $\mathcal{S} S p_{\mathcal{O}, c}$, and hence that any Reedy fibrant Segal category is fibrant in the appropriate $\mathcal{L} \mathcal{S} S p_{\mathcal{O}, c}$. The following proposition shows that the converse statement holds as well.

Proposition 3.1. A fibrant object in $\mathcal{S} S p_{\mathcal{O}, c}$ is fibrant in the Reedy model structure on simplicial spaces. In particular, a fibrant object of $\mathcal{L} \mathcal{S} \mathcal{S} p_{\mathcal{O}, c}$ is fibrant in the Reedy structure.

Proof. Let $W$ be a fibrant object in $\mathcal{S} S p_{\mathcal{O}, c}$. We need to show that the map $W \rightarrow$ $\Delta[0]^{t}$ has the right lifting property with respect to all levelwise acyclic cofibrations, not just the ones in $\mathcal{S} \mathcal{S} p_{\mathcal{O}, c}$. We first consider the case where $A \rightarrow B$ is an acyclic cofibration where $A$ and $B$ are Segal precategories, say in $\mathcal{S} S p_{\mathcal{O}^{\prime}, c}$ for some set $\mathcal{O}^{\prime} \neq \mathcal{O}$.

Using the 0 -skeleta $\operatorname{sk}_{0}(A)$ and $\operatorname{sk}_{0}(W)$, we can define a simplicial space $A^{\prime}$ as the pushout

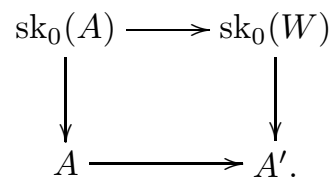

Note in particular that the induced map $A_{0}^{\prime} \rightarrow W_{0}$ is an isomorphism. Now, we can define $B^{\prime}$ as a pushout

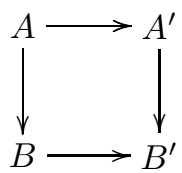


and thus a diagram

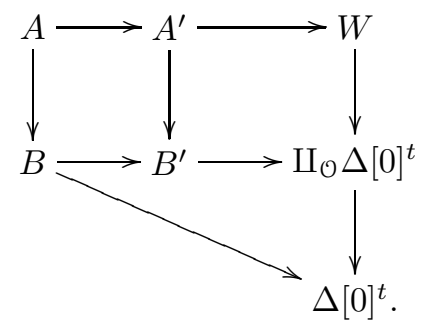

Because it is defined as a pushout along an acyclic cofibration in the Reedy structure, the map $A^{\prime} \rightarrow B^{\prime}$ is also an acyclic cofibration, and $A_{0}^{\prime} \cong B_{0}^{\prime} \cong \amalg_{\mathcal{O}} \Delta[0]^{t}$. Therefore there exists a lift $B^{\prime} \rightarrow W$, from which there exists a lift $B \rightarrow W$.

Now, suppose that $A \rightarrow B$ is an acyclic cofibration between simplicial spaces that are not necessarily Segal precategories. Since $W$ and $\Delta[0]^{t}$ are Segal precategories, we can factor our diagram as

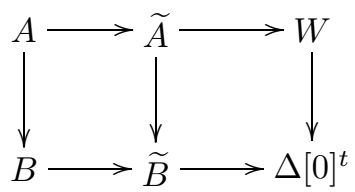

where $\widetilde{A}$ and $\widetilde{B}$ are obtained from $A$ and $B$, respectively, by collapsing the space in degree zero to its components. Then, we obtain a lifting from the previous argument.

Since fibrant objects in $\mathcal{L} \mathcal{S} \mathcal{S} p_{\mathcal{O}, c}$ are fibrant in $\mathcal{S} \mathcal{S} p_{\mathcal{O}, c}$, the second statement of the proposition follows as well.

Using this result, we turn to the converse to Corollary 2.2 .

Theorem 3.2. Any Reedy fibrant Segal category is fibrant in Se at $_{c}$.

Proof. Let $W$ be a Reedy fibrant Segal category and suppose that $f: A \rightarrow B$ is a generating acyclic cofibration in $\mathcal{S} e \mathrm{C} a t_{c}$. We need to show that the map $W \rightarrow \Delta[0]^{t}$ has the right lifting property with respect to the map $f$. We know that it has the right lifting property with respect to any such $f$ which preserves a fixed object set $\mathcal{O}$, by Proposition 3.1. Therefore, we assume that $f$ is a monomorphism but is not surjective.

Choose a $b \in B_{0}$ that is not in the image of $f: A \rightarrow B$. Since $f$ is a weak equivalence, we know that $b$ is equivalent in $L_{c} B$ to $f(a)$ for some $a \in\left(L_{c} A\right)_{0}=A_{0}$. Define $\left(L_{c} B\right)_{a}$ to be the full Segal subcategory of $L_{c} B$ whose objects are in the essential image of $a$. Let $B_{a}$ be the sub-simplicial space of $B$ whose image is in $\left(L_{c} B\right)_{a}$. Note that $\left(B_{a}\right)_{0}=\left(\left(L_{c} B\right)_{a}\right)_{0}$.

Now, define $A_{a}$ to be the Segal precategory having as 0 -space the union of $\left(B_{a}\right)_{0}$ and $A_{0}$ and for which

$$
\left(A_{a}\right)_{n}\left(a_{0}, \ldots, a_{n}\right)=A_{n}(a, \ldots, a)
$$


for all $a_{i} \in\left(A_{a}\right)_{0}$. Letting $a$ also denote the doubly constant simplicial space given by $a$, we can then define $A_{1}$ to be a pushout given by

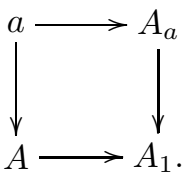

Notice that the map $A \rightarrow A_{1}$ has a section and that we can factor $f$ as the composite $A \rightarrow A_{1} \rightarrow B$.

We now repeat this process by choosing a $b^{\prime}$ which is not in the image of the map $A_{1} \rightarrow B$ and a corresponding $a^{\prime}$, and continue to do so, perhaps infinitely many times, and take a colimit to obtain a Segal precategory $\widehat{A}$ such that the map $f$ factors as $A \rightarrow \widehat{A} \rightarrow B$ and there is a section $\widehat{A} \rightarrow A$. Furthermore, notice that $\widehat{A}_{0}=B_{0}$ and that the map $\widehat{A} \rightarrow B$ is object-preserving. Notice also that it is an acyclic cofibration. Therefore, the dotted-arrow lift exists in the following diagram:

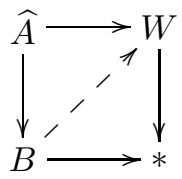

which implies, using the section $\widehat{A} \rightarrow A$, that there is a dotted arrow lift in the diagram

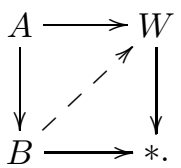

Note. One might wonder why, in the general case, the model category structure $\mathrm{SeC} a t_{c}$ is not defined as a localization of a model structure with object-preserving weak equivalences, as it is in the fixed object set situation. We prove in [2, 3.12] that it is impossible, simply because the more basic model structure cannot exist. Therefore, we cannot use the tools of localized model structures. Much of the difficulty with working with $\mathcal{S} e \mathrm{C} a t_{c}$ arises from this fact.

\section{Projective fibrant Segal categories}

In this section, we show that the analogous statement holds in our other model category structure, $\mathcal{S} e \mathcal{C} a t_{f}$. In this structure, the weak equivalences are the same as those of $\mathcal{S} e \mathrm{C} a t_{c}$, but the fibrations and cofibrations are defined differently. They are technical to describe, so we will refer the interested reader to $2, \S 4,7.1$ ] for a complete description. The fibrations should be thought of heuristically as those we would obtain via a localization of a model structure given by levelwise weak equivalences and fibrations, although, as mentioned at the end of the previous section, such a construction is impossible in the general case.

As before, we begin by considering the special case where we have a fixed set $\mathcal{O}$ in degree zero. Analogously to the situation in the previous section, there exists a model category structure $\mathcal{S} \mathcal{S} p_{\mathcal{O}, f}$ on simplicial spaces with a fixed set $\mathcal{O}$ in degree zero in which the weak equivalences and fibrations are levelwise. Localizing this 
model structure with respect to a set of maps results in a model structure $\mathcal{L} \mathcal{S} S p_{\mathcal{O}, f}$ in which the fibrant objects are Segal categories with $\mathcal{O}$ in degree zero [1,3.8].

We can prove the following result using the same techniques that we used to prove Proposition 3.1

Proposition 4.1. The fibrant objects in $\mathcal{L} \mathcal{S} \mathcal{S} p_{\mathcal{O}, f}$ are precisely the Segal categories that have the set $\mathcal{O}$ in degree zero and are fibrant in the projective model structure on simplicial spaces.

As in the Reedy case, there is a corresponding functor $L_{f}$ (where "Segal spaces" are now obtained as a localization in the projective, rather than the Reedy, model structure), and in [2, §7] we show that the weak equivalences are actually independent of whether we define them in terms of $L_{c}$ or $L_{f}$.

Theorem 4.2. Segal categories that are fibrant in the projective model category structure on simplicial spaces are fibrant in $\mathrm{SeCat}$. $_{\text {. }}$.

Proof. The argument given for Selat $t_{c}$ still holds in this case.

\section{ACKNOWLEDGMENTS}

I would like to thank Bertrand Toën for pointing out an error in a previous proof of the main result, and the referee for suggestions which led to an improved proof of Proposition 3.1

\section{REFERENCES}

[1] J.E. Bergner, Simplicial monoids and Segal categories, Contemp. Math. 431, 2007, 59-83.

[2] J.E. Bergner, Three models for the homotopy theory of homotopy theories, Topology 46 (2007), 397-436.

[3] W.G. Dwyer, D.M. Kan, and J.H. Smith, Homotopy commutative diagrams and their realizations. J. Pure Appl. Algebra 57(1989), 5-24. MR984042 (90d:18007)

[4] W.G. Dwyer and J. Spalinski, Homotopy theories and model categories, in Handbook of Algebraic Topology, Elsevier, 1995. MR1361887 (96h:55014)

[5] P.G. Goerss and J.F. Jardine, Simplicial Homotopy Theory, Progress in Math, vol. 174, Birkhäuser, 1999. MR 1711612 (2001d:55012)

[6] Philip S. Hirschhorn, Model Categories and Their Localizations, Mathematical Surveys and Monographs 99, Amer. Math. Soc., 2003. MR.1944041 (2003j:18018)

[7] A. Hirschowitz and C. Simpson, Descente pour les $n$-champs, preprint available at math.AG/9807049.

[8] Mark Hovey, Model Categories, Mathematical Surveys and Monographs, 63. American Mathematical Society, 1999. MR:1650134(99h:55031)

[9] A. Joyal, The theory of quasi-categories, in preparation.

[10] André Joyal and Myles Tierney, Quasi-categories vs. Segal spaces, Contemp. Math. 431, 2007, $277-325$.

[11] C.L. Reedy, Homotopy theory of model categories, unpublished manuscript, available at http://www-math.mit.edu/ ${ }^{\text {psh. }}$

[12] Charles Rezk, A model for the homotopy theory of homotopy theory, Trans. Amer. Math. Soc., 353 (2001), no. 3, 973-1007. MR1804411(2002a:55020)

[13] Carlos Simpson, A closed model structure for $n$-categories, internal Hom, $n$-stacks, and generalized Seifert-Van Kampen, preprint, available at math.AG/9704006. MR 1141208 (92m:14014)

[14] Z. Tamsamani, Sur des notions de $n$-catégorie et $n$-groupoïde non strictes via des ensembles multi-simpliciaux, preprint available at alg-geom/9512006. $K$-theory 16 (1999), 51-99. MR:1673923 (99m:18007)

Kansas State University, 138 Cardwell Hall, Manhattan, Kansas 66506

E-mail address: bergnerj@member.ams.org 